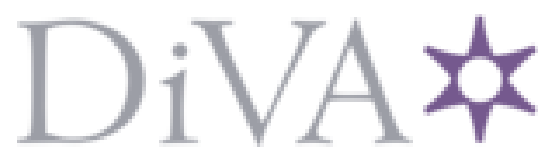

http://www.diva-portal.org

\title{
Preprint
}

This is the submitted version of a paper published in Science.

Citation for the original published paper (version of record):

Trinajstic, K., Sanchez, S., Dupret, V., Tafforeau, P., Long, J. et al. (2013)

Fossil Musculature of the Most Primitive Jawed Vertebrates.

Science, 341 (6142): 160-164

http://dx.doi.org/10.1126/science.1237275

Access to the published version may require subscription.

N.B. When citing this work, cite the original published paper.

Permanent link to this version:

http://urn.kb.se/resolve?urn=urn:nbn:se:uu:diva-206443 


\title{
Title: Fossil musculature of the most primitive jawed vertebrates.
}

Authors: Kate Trinajstic ${ }^{1,2}$, Sophie Sanchez ${ }^{3,4}$, Vincent Dupret ${ }^{4}$, Paul Tafforeau ${ }^{3}$, John Long ${ }^{5,6}$, Gavin Young ${ }^{6}$, Tim Senden ${ }^{7}$, Catherine Boisvert ${ }^{8}$, Nicola Power ${ }^{6}$, Per Erik Ahlberg ${ }^{4 *}$

\begin{abstract}
Affiliations:
${ }^{1}$ Western Australian Organic and Isotope Geochemistry Centre, Department of Chemistry, Curtin University, Perth, Western Australia 6102, Australia.

${ }^{2}$ Earth and Planetary Sciences, Western Australian Museum, Perth, Western Australia 6000, Australia.

${ }^{3}$ European Synchrotron Radiation Facility, 38043 Grenoble Cedex, France.

${ }^{4}$ Subdepartment of Evolution and Development, Department of Organismal Biology, Uppsala University, Norbyvägen 18A, 75236 Uppsala, Sweden.

${ }^{5}$ School of Biological Sciences, Flinders University, PO Box 2100, Adelaide, South Australia 5001 .

${ }^{6}$ Research School of Earth Sciences, The Australian National University, Canberra 0200, Australia.

${ }^{7}$ Department of Applied Mathematics, Research School of Physics and Engineering, The Australian National University, Canberra 0200, Australia.

${ }^{8}$ Australian Regenerative Medicine Institute, Monash University, Victoria 3800, Australia.
\end{abstract}

*Correspondence to: Per Erik Ahlberg, Per.Ahlberg@ebc.uu.se

Abstract: The transition from jawless to jawed vertebrates (gnathostomes) resulted in the reconfiguration of the muscles and skeleton of the head, including the creation of a separate shoulder girdle with distinct neck muscles. We describe here the only known examples of preserved musculature from placoderms (extinct armored fishes), the phylogenetically most basal jawed vertebrates. Placoderms possess a regionalized muscular anatomy differing radically from the musculature of extant sharks, which is often viewed as primitive for gnathostomes. The placoderm data suggest that neck musculature evolved together with a dermal joint between skull and shoulder girdle, not as part of a broadly flexible neck as in sharks, and that transverse abdominal muscles are an innovation of gnathostomes rather than of tetrapods.

\section{Main Text:}

The most fundamental anatomical divide among living vertebrates is that between the jawless cyclostomes (lampreys and hagfishes) and the jawed gnathostomes (all other vertebrates). Although early development in the two groups is similar, differences in migration and 
proliferation patterns of cell populations produce profoundly different adult architectures in the head region, encompassing not only the skeleton but also the musculature (1). In conjunction with the presence or absence of jaws, a major difference between the groups is the absence of a shoulder girdle, paired appendages, and a differentiated neck region in cyclostomes.

The gnathostome stem group contains jawless as well as jawed clades, suggesting the jawless condition is primitive for vertebrates (2). Some jawless stem gnathostomes, such as osteostracans, have pectoral fins and "shoulder girdles", but the latter are immovably attached to the skull (2). Head-shoulder separation, and the origin of distinctive neck muscles such as the cucullaris, seems to have occurred at approximately the same time as the origin of jaws. The phylogenetically deepest and morphologically most primitive versions of jawed vertebrate anatomy are found in the placoderms, a paraphyletic array of Silurian-Devonian armored fishes that form the upper part of the gnathostome stem group $(3,4)$ (Fig. 1A).

Data on placoderm muscular anatomy would add considerably to the knowledge of gnathostome origins, given the functional and anatomical importance of the musculature in the transition from jawless to jawed vertebrates, as well as its significance as a probable indicator of cell population identity (5). Until now, such data have not been available, and conclusions about placoderm musculature have been based entirely on inferences from skeletal anatomy and functional morphology. Studies of muscular evolution in gnathostomes have often proceeded from the assumption that extant sharks are adequate models for the primitive gnathostome condition $(6,7,8)$. 
We present here placoderm fossils with preserved three-dimensional musculature, from the Upper Devonian Gogo Formation of Western Australia. The presence of neck and trunk muscles in the eubrachythoracid arthrodires Eastmanosteus, Compagopiscis and Incisoscutum allow detailed reconstruction of this musculature (Figs 1B to D; 2 and 3; and figs S1 and S2). Additional information about tail muscles comes from the ptyctodont Austroptyctodus (fig. S2H to I). These specimens present an anatomy that differs radically from the shark model (6).

Presence of a dermal neck joint (Fig. 2A) between the skull and trunk armor of placoderms implies vertical pivoting movement of the head, requiring elevator and depressor muscles $(9,10)$. Such muscles are confirmed in our specimens (Figs $1 \mathrm{~B}$ to $\mathrm{C} ; 3 \mathrm{~A}$ to $\mathrm{F}$ and $\mathrm{J}$ and fig. S1). A large medial levator capitis major and a small lateral levator capitis minor have palmately arranged muscle fibres radiating from the area of origin. Lateral to the articulation of the neck joint (Fig. 2A), an oblique muscle that originates on the inner face of the dermal shoulder girdle and inserts in a hollow on the inner face of the skull roof is identified as the cucullaris muscle (see Supplementary text 1 and fig. S1F). It represents the phylogenetically deepest record of this characteristic gnathostome muscle. In mammals the cucullaris reaches the dorsal midline of the neck, whereas in sharks it is narrower and bounded dorsally by somitic epaxial muscles. The latter morphology is often viewed as primitive for gnathostomes (11), but both differ from the placoderm condition in forming part of a flexible neck region rather than operating a constrained pivoting joint.

The cranial musculature is less well preserved. However, the levator arcus palatini (11) is observed attached to the ventral postocular process of Compagopiscis (Fig. 3K to L; fig. S2G 
and Supplementary movie). In addition, part of the dorsal branchial constrictor muscle can be seen on the visceral surface of the paranuchal plate, lateral to the cucullaris (fig. S1J).

Normal segmented body muscles with anteroposteriorly oriented fibers are present under the dorsolateral parts of the trunk armor and in the tail. However, the posterior part of the ventral abdominal musculature contains two anteroposteriorly elongated muscle bands with transversely oriented fibers (Figs $1 \mathrm{C}$ to $\mathrm{D}$ and $2 \mathrm{C}$ to $\mathrm{F}$ see supplementary information 2), representing areas of stronger fiber development within a continuum of transverse fibers extending across the ventral midline. Dorsolaterally, the bands are in contact with the ventral ends of the myomeres. The existence of these transverse muscles had not been predicted (9).

Mineralized tendinous attachments for the somitic muscles, inclined anterodorsally in a manner reflecting the $\mathrm{W}$ shape of the myocommata, are present in the median septum above the neural spines (Fig. 2B). In the eubrachythoracids, the myocommata are incompletely preserved (Fig. 3D to I and fig. S2F), but in Austroptyctodus they have a shallow w-shaped curvature (12) (fig. S2H to I). The medial myoseptal attachment probably spanned only two segments, in contrast to at least three segments in modern chondrichthyans and teleosts (13). In extant gnathostomes the connections between myosepta and skin are biomechanically important $(13,14)$; preserved Incisoscutum skin contains obliquely oriented tendons similar to those in sharks (fig. S2C to E), suggesting a similar role for the skin as a store of elastic energy $(13,14)$.

The musculature of eubrachythoracid arthrodires, characterized by shallow myoseptal curvature, differentiated head elevators positioned as antagonists to the cucullaris, and bands of transverse- 
fiber muscles in the abdomen, differs greatly from that of sharks. The latter is dominated by deeply W-shaped myomeres, lacks transverse abdominal muscles, and includes a broadly flexible neck region with a narrow triangular cucullaris forming a dorsal edge to the branchial chamber $(11,13)$. Most of the differences appear to reflect the overall contrast between a relatively uniform, flexible body in sharks and a regionalized body with a pivoting neck joint and rigid trunk armor in arthrodires. Their evolutionary significance hinges on whether eubrachythoracid musculature is specialized or primitive relative to that of sharks. Placoderms appear to be a paraphyletic segment of the gnathostome stem group $(3,4)$, so if any components of eubrachythoracid musculature can be shown to be general for placoderms they can also be inferred to be primitive relative to the crown group. The status of the shallow myoseptal curvature cannot yet be determined in this regard, but the muscles of the neck joint and abdomen have specific skeletal associations that allow such phylogenetic inferences to be drawn.

Most ostracoderms, a grade of jawless stem gnathostomes (2) (Fig. 1A), have 'head shields' that also encompass the shoulder girdle region (2). This suggests that the gnathostome shoulder girdle originated through subdivision of the shield. Almost all placoderms have a mobile joint between the skull and shoulder girdle, implying the need for elevator and depressor muscles like those observed in eubrachythoracids. A cucullaris operating this joint, antagonistic to specialized epaxial head elevators, is thus probably primitive relative to the crown gnathostome condition of a cucullaris without specialized antagonists that forms part of a broadly flexible neck.

The transverse abdominal muscles of eubrachythoracids are not as directly tied to a skeletal structure with an identifiable mechanical function. Comparison with recent elephant shark 
indicates that they are not homologous with any muscles of the pelvic fin or male clasper (Supplementary text 2). However, they may modulate shear forces between the armor and laterally undulating body during tail-propelled swimming. A long ventral armor is also present in antiarchs, recovered as the most primitive placoderms in several recent analyses $(3,4,15)$. Transverse abdominal muscles may thus be an attribute of the placoderm segment of the gnathostome stem group, and hence primitive relative to the absence of such muscles at the base of the gnathostome crown group.

Outside of placoderms, transversely oriented abdominal muscle fibers are restricted to tetrapods and have been regarded as a tetrapod autapomorphy (16). Their associated connective tissues and tendons, which are derived from the somatopleure component of the lateral plate mesoderm (17), play an important role in hypaxial myogenesis (18). In lampreys, the posterior lateral plate mesoderm is not separated into splanchnic and somatopleuric components (19), meaning that it cannot give rise to somatopleure-derived structures such as paired fins. The presence of paired fins in placoderms shows that separation of somatopleure and splanchnopleure had occurred, supporting the inference that their transverse muscles may have been patterned by the same somatopleure-based mechanism as in tetrapods.

The arthodires of the Gogo Formation reveal an elaborate regionalized musculature including the earliest and phylogenetically deepest examples of several muscle types. Particularly surprising is the extensive development of transverse-fibre muscles in the ventral body wall, which parallels the condition in tetrapods. Hypothetical reconstructions are not able to recover the full complexity of this musculature, either on the basis of biomechanical analysis or phylogenetic 
bracketing, and are thus liable to give a false picture of muscular evolution at the origin of gnathostomes. The study of exceptionally preserved fossils will continue to provide essential data for the reconstruction of vertebrate soft anatomy, particularly in groups with no close living relatives.

\section{References and Notes:}

1.Y. Oisi, K. G. Ota, S. Kuraku, S. Fujimoto, S. Kuratani. Craniofacial development of hagfishes and the evolution of vertebrates. Nature 493, 175-180 (2013).

2. P. Janvier, Early Vertebrates (Oxford Univ. Press, 1996).

3. M. D. Brazeau. The braincase and jaws of a Devonian 'acanthodian' and modern gnathostome origins. Nature 457, 305-308 (2009).

4. S. P. Davis, J. A. Finarelli, M. I. Coates. Acanthodes and shark-like conditions in the last common ancestor of modern gnathostomes. Nature 486, 247-250 (2012).

5. T. Matsuoka, P. E. Ahlberg, N. Kessaris, P. Iannarelli, U. Dennehy, W. D. Richardson, A. P. McMahon, G. Koentges. Neural crest origins of the neck and shoulder. Nature 436, 348-355 (2005).

6. J. Mallatt. Ventilation and the origin of jawed vertebrates: a new mouth. Zool. J. Linn. Soc. 117, 329-404 (1996).

7. S. Kuratani. Evolution of the vertebrate jaw: comparative embryology and molecular developmental biology reveal the factors behind evolutionary novelty. J. Anat. 205, 335-347 (2004).

8. S. Kuratani. Evolutionary developmental studies of cyclostomes and the origin of the vertebrate neck. Dev. Growth Diff. 50, S189-S194 (2008). 
9. A. Heintz, in The Bashford Dean Memorial Volume: Archaic Fishes E. W. Gudger, Ed.

(American Museum of Natural History, 1930). pp.115-224.

10. R. Miles, T. S. Westoll. The placoderm fish Coccosteus cuspidatus Miller ex Agassiz, from the Middle Old Red Sandstone of Scotland. Part 1. Descriptive morphology. Trans. R. Soc. of Edinb 67, 373-476 (1968).

11. F. H. Edgeworth, The Cranial Muscles of Vertebrates (Cambridge Univ. Press, 1935).

12. K. Trinajstic, C. Marshall, J. Long, K. Bifield. Exceptional preservation of nerve and muscle tissues in Late Devonian placoderm fish and their evolutionary implications. Biol. Lett. 3, 197200 (2007).

13. S. Gemballa, L. Ebmeyer, K. Hagen, T. Hannich, K. Hoja, M. Rolf, K. Treiber, F. Vogel, G. Weitbrecht. Evolutionary transformations of myoseptal tendons in gnathostomes. Proc. R. Soc. Lond. B 270, 1229-1235 (2003).

14. S. A. Wainright, F. Vosburgh, J. H. Hebrank. Shark skin: function in locomotion. Science 202, 747-749 (1978).

15. M. Zhu, X. Yu, B. Choo, J. Wang, L. Jia. An antiarch placoderm shows that pelvic girdles arose at the root of jawed vertebrates. Biol. Lett. 8, 453-456 (2012).

16. N. Schilling. Evolution of the axial system in craniates: morphology and function of the perivertebral musculature. Front. Zool. 8, 4 (2011).

17. B. Christ, M. Jacob, H. J. Jacob. On the origin and development of the ventrolateral abdominal muscles in the avian embryo. Anat Embryol. 166, 87-101 (1983).

18. S. J. Mathew, J. M. Hansen, A. J. Merrel, M. M. Murphy, J. A. Lawson, D. A. Hutcheson, M. S. Hansen, M. Angus-Hill, G. Kardon. Connective tissue fibroblasts and Tcf4 regulate myogenesis. Development, 138, 371-384 (2011). 
19. K. Onimaru, E. Shoguchi, S. Kuratani, M. Tanaka. Development and evolution of the lateral plate mesoderm: comparative analysis of amphioxus and lamprey with implications for the acquisition of paired fins. Dev. Bio. 359,124-136, (2011).

20. D. Paganin, S. C. Mayo, T. E. Gureyev, P. R. Miller, S. W. Wilkins. Simultaneous phase and amplitude extraction from a single defocused image of a homogeneous object. J. Microsc. 206, $33-40$ (2002).

21. S. Sanchez, P. E Ahlberg, K. M. Trinajstic, A. Mirone, P. Tafforeau.Three-dimensional synchrotron virtual paleohistology: a new insight into the world of fossil bone microstructures. Microsc. Microanal.18, (5) 1095-1105 (2012).

22. T. Varslot, A. Kingston, G. Myers, A. Sheppard. High-resolution helical cone-beam microCT with theoretically-exact reconstruction from experimental data. Medical Physics 38 (10), 5459-5476 (2011).

23. P. S. L. Anderson, M.W. Westneat. Feeding mechanics and bite force modelling of the skull of Dunkleosteus terrelli, an ancient apex predator. Biol. Lett. 3, 76-79 (2007).

24. Z. Johanson. Placoderm branchial and hypobranchial muscles and origins in jawed vertebrates. J. Vertebr. Paleontol. 23, 735-749 (2003).

25. G. M. Couly, P. M. Coltey, N.M Le Douarin. The triple origin of skull in higher vertebrates: a study in quail-chick chimaeras. Development 117, 409-429. (1993).

26. R. Huang, Q. Zhi, C. P. Ordahl, B. Christ. The fate of the first avian somite. Anat. Embryol. 195, 435-449 (1997).

27. Huang, Q. Zhi, K. Patel, J. Wilting, B. Christ. Contributions of single somites to the skeleton and muscles of the occipital and cervical regions in avian embryos. Anat. Embryol. 202, 375-383 (1997). 
28. D. M. Noden. Embryonic origins of avian cephalic and cervical muscles and associated connective tissue. Am. J. Anat. 168, 257-276 (1983).

29. S.Theis, K. Patel, et al. The occipital lateral plate mesoderm is a novel source for vertebrate neck musculature. Development 137(17), 2961-2971 (2010).

30. P. E. Ahlberg, K. M. Trinajstic, J. A. Long, Z. Johanson. Pelvic claspers confirm chondrichthyan-like internal fertilization in arthrodires. Nature 460, 888-889 (2009).

We acknowledge M. Siversson at the Western Australian Museum, Perth and Z. Johanson at the Natural History Museum, London for lending us specimens in their care. We thank I. Montero Verdú for his picture of the muscle bundles (Fig. 3d) and Alex Ritchie for an Eastmanosteus image. K. Trinajstic, P. E. Ahlberg \& C. Boisvert are supported by an ARC QEII Fellowship DP110101127 and J. Long, K. Trinajstic, T. Senden \& G.Young by ARC DP 1092870. S. Sanchez, V. Dupret \& P. E. Ahlberg are supported by ERC Advanced Investigator Grant 233111, P. E. Ahlberg by a Wallenberg Scholarship from the Knut and Alice Wallenberg Foundation, and C Boisvert is supported by a Human Frontiers Research Program and an ARC Discovery project DP 1096002. The scan performed at the European Synchrotron Radiation Facility in Grenoble, France, was part of project EC770. K. Trinajstic acknowledges the 2010 Prime Minister's Science Prize and J. Long acknowledges funding from The Australian Geographic Society which supported fieldwork at Gogo. Specimens are housed in the collections of the Western Australian Museum (WAM), Australian National University (ANU), Australian Museum (AMF) and Museum Victoria (MV), Australia, and the Natural History Museum (NHM), UK. 
Fig. 1. Preserved musculature and placoderm phylogeny. A, Phylogeny showing position of placoderms relative to major extant vertebrate groups. Placoderm paraphyly indicated schematically by inclusion of two groups, antiarchs and arthrodires. Topology from references $(3,4)$. B-D, Preserved musculature of eubrachythoracid placoderms.B, dorsal, C, lateral, and D, ventral views. Muscle fiber orientations indicated diagrammatically. Scale bar, $10 \mathrm{~mm}$.

Fig. 2. Dermal skeleton, mineralized tendons and transverse abdominal muscles. A, Eastmanosteus (AMF82185) lateral view showing dermal neck joint (indicated by red ring). B, Incisoscutum (WAM 03.3.28), mineralized tendinous attachments. C, Incisoscutum (NHM P57636A) dorsal view; red box enlarged in E. D, Incisoscutum (WAM 03.3.24), in ventral view showing body cavity infill; red box enlarged in F. E, transverse and longitudinal abdominal muscles in internal view (NHM P 57636A). F, corresponding muscles in external view (WAM 03.3.24). Scale bars for A-D, $10 \mathrm{~mm}$; for $\mathbf{E}-\mathbf{F}, 1 \mathrm{~mm}$. ADL, anterior dorsolateral plate; AL, anterior lateral plate; AVL, anterior ventrolateral plate; df, dorsal fin; lf, longitudinal muscle fibers; MD, median dorsal plate; MV, median ventral plate; mineralized tendons; nsp, neural spine; $\mathrm{Nu}$, nuchal plate; mit, $\mathrm{PNu}$, paranuchal plate; PVL, posterior ventrolateral plate; tf, transverse muscle fibers.

Fig. 3. Muscle Preservation. A, Eastmanosteus (ANU V2582) in dorsal view showing the nuchal gap. B, Detail ANU V2582. C, Compagopiscis (WAM 11.12.05) in dorsal view, showing levator capitis major and minor. D, Incisoscutum (WAM 10.01.02), levator capitis major and segmented trunk muscles underlying median dorsal plate, dorsolateral view. E, F, Incisoscutum (WAM 95.1.1), internal view of median dorsal plate with muscle fibers. F, 
magnification of red box in E. G-I, Incisoscutum (NHM P50934). G, vertebral elements below median dorsal plate. H, Proposed horizontal myoseptum. I, Trunk muscles. J, Cucullaris muscle of Compagopiscis (WAM 11.12.05), attached to the internal surface of the anterior dorsolateral plate. K, L, Compagopiscis (WAM 12.8.1), showing levator arcus palatini attaching to the left ventral postocular process. $\mathbf{K}$, lateral view. $\mathbf{L}$, posteroventral view. Scale bars for A-B, $10 \mathrm{~mm}$; for C-L, $1 \mathrm{~mm}$. Abbreviations as for Fig. 2, plus: art con, articular condyle of neck joint; cuc, cucullaris; ?hs, possible horizontal septum; hsp, haemal spine; lap, levator arcus palatini; lcma, levator capitis major; lcmi, levator capitis minor; mc, myocomma; MDk, median dorsal keel; mf, muscle fibers; Nu gap, nuchal gap; om, orbital margin; sm, segmented muscle block; ve, vertebral elements.

\section{Supplementary Materials:}

Materials and Methods

Supplementary Text 1

Supplementary Text 2

Figures S1-S2

Movies S1

References (21-24) 
The gnathostome crown group

A

\section{Cyclostomata}

no paired fins

no transverse abd. muscles

unknown

\section{Placodermi}

paired fins paired fins

transverse

abd.muscles

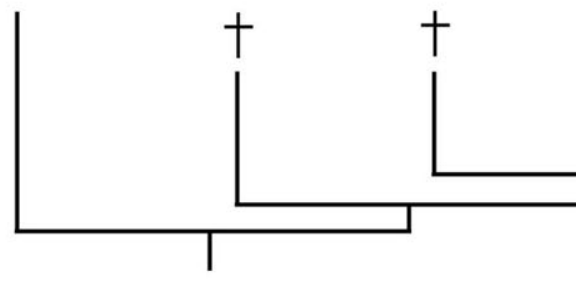

\section{Chondrichthyes}

paired fins

no transverse

abd. muscles

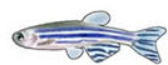

\section{paired fins}

no transverse

\section{Actinistia, Dipnoi}

paired fins

no transverse abd. muscles

\section{Tetrapoda}

paired limbs

transverse abd. muscles
B

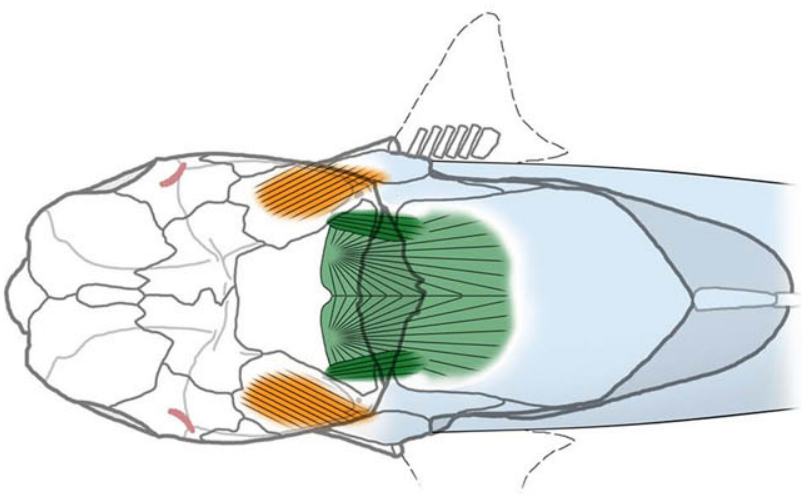

cucullaris

levator arcus palatini

visceral surface of bone; orbit

C

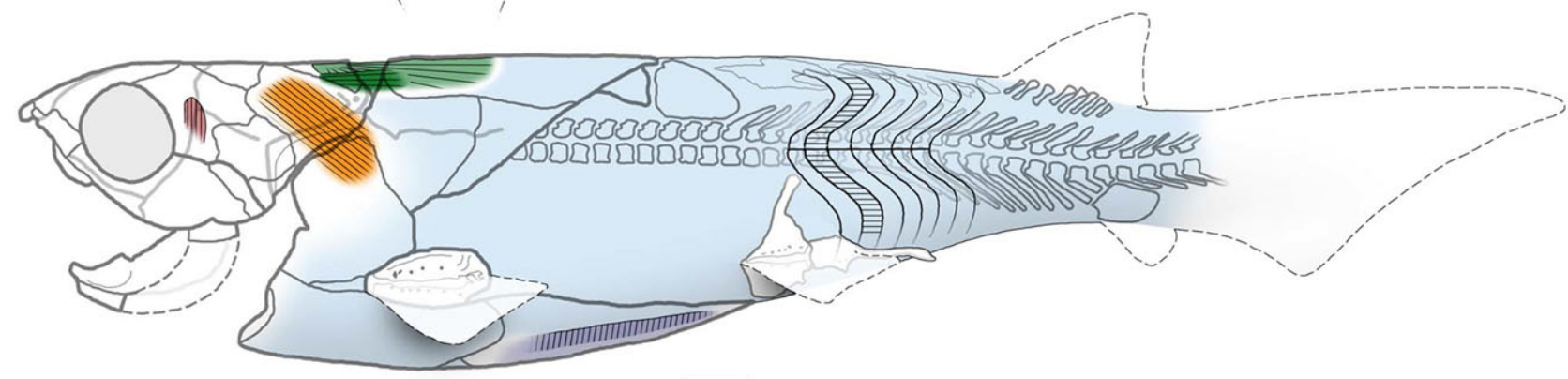

D

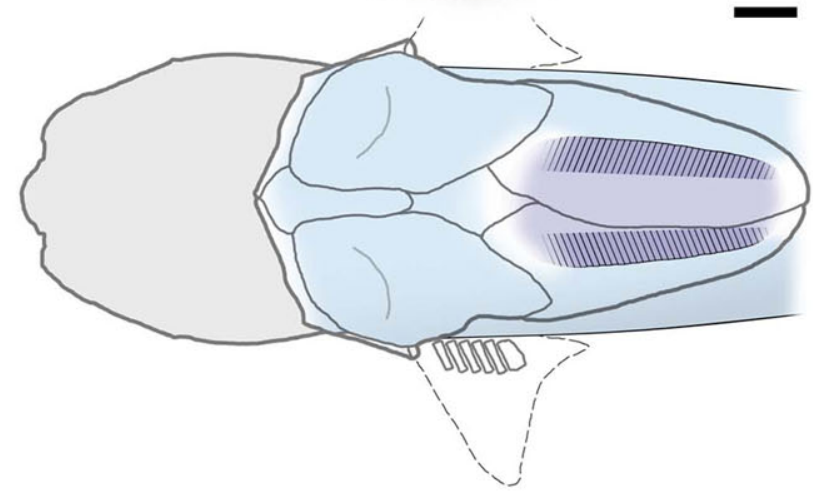

segmented body musculature

levator capitis major

levator capitis minor

transversus abdominis

area of faint transverse muscle fibres 


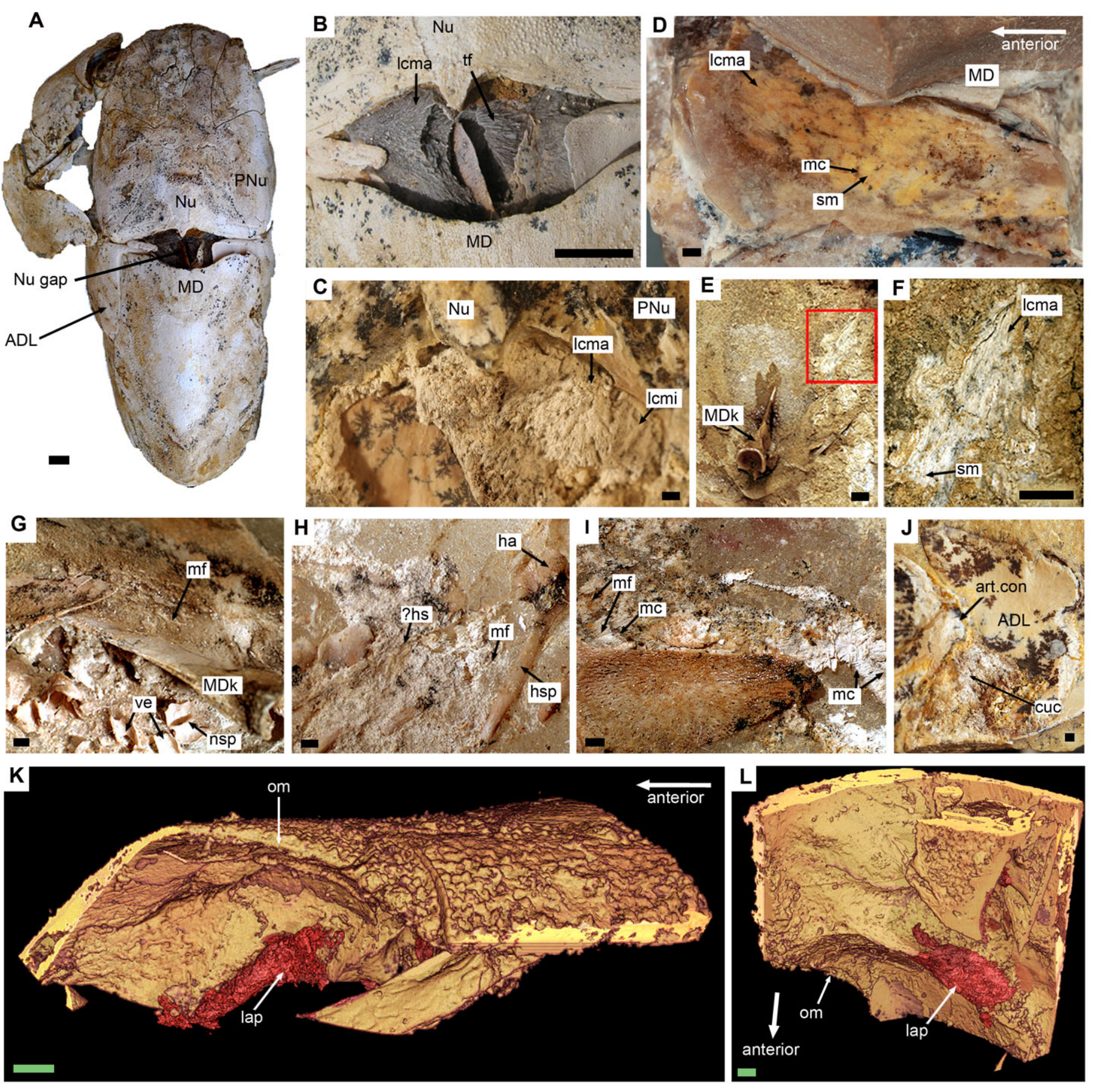




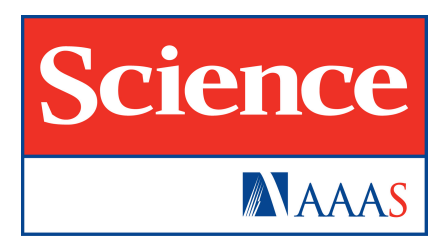

\section{Supplementary Materials for}

\section{Fossil Musculature of the Most Primitive Jawed Vertebrates}

Kate Trinajstic, Sophie Sanchez, Vincent Dupret, Paul Tafforeau, John Long, Gavin

Young, Tim Senden, Catherine Boisvert, Nicola Power, Per Erik Ahlberg

correspondence to: per.ahlberg@ebc.uu.se

\section{This PDF file includes:}

Materials and Methods

SupplementaryText

Figs. S1 to S4

Caption for Movie S1

Other Suplementary Materials for this manuscript includes the following:

Movie S1 


\section{Materials and Methods}

All fossil specimens were originally collected from the Gogo Formation, Western Australia. Preparation of Eastmanosteus followed the standard acid etching technique using dilute formic acid, whereas acetic acid $(<10 \%)$ was used in the preparation of Compagopiscis and Incisoscutum. Compagopiscis (WAM12.8.1) was scanned at beamline ID19, European Synchrotron Radiation Facility (ESRF, Grenoble France) using propagation phase contrast $(4 \mathrm{~m})$. The specimen was imaged using a white beam filtered with $9 \mathrm{~mm}$ of copper and $2 \mathrm{~mm}$ of aluminium, using a W150 wiggler with the gap set to $41 \mathrm{~mm}$. The resulting spectrum has an average energy of $131 \mathrm{keV}$, well adapted to scanning such a large sample. We used a $125 \mu \mathrm{m}$ thick LuAG:Ce crystal (Lutecium Aluminium Garnet doped with Cerium) scintillator and a FReLoN (Fast Readout Low Noise) 2K14 CCD camera mounted on an optic delivering a pixel size of $7.45 \mu \mathrm{m}$. The scan was performed in half-acquisition, continuous mode, over 360 degrees, with 5000 projections. The exposure time was $0.1 \mathrm{~s}$. The data were reconstructed using a single distance phase retrieval algorithm $(20,21)$ to maximize the contrast of the structures in the data. The segmentation of the data was done using VGStudio Max 2.2 (Volume Graphics, Germany).

Micro-core samples of Compagopiscis WAM (03.3.24) were imaged with a microCT system build at ANU (22) using a $80 \mathrm{kV} / 100$ microA transmission source focused for 2 micron radiographic resolution and a $1 \mathrm{~mm}$ Al filter. Specimens were photographed either with a Canon 550D and a Canon 100mm macro lens IS 2.8, or with a Canon20D camera and Canon 100mm macro lens and Leica microscope using Firecam software. Specimens which were resin-embedded or where muscle was embedded in calcite matrix were photographed through a layer of alcohol. Specimens of Callorhinchus milii (Elephant shark) were obtained from Western Port Bay, Victoria by hook and line (Permit number RP1003; ethics approval MAS/ARMI/2010/01 and were skinned to study muscle arrangements.

\section{Supplementary Text}

Head and neck musculature in placoderms

Detailed inferences about placoderm musculature have been derived from functional arguments and preserved muscle scars on dermal bones (23,24). However, while these inferences are well founded they do not amount to a sufficient description of placoderm musculature. Head elevator muscles, previously hypothesised for the eubrachythoracids Dunkleosteus ("Dinichthys") and Coccosteus $(9,10,23)$, are well preserved in our eubrachythoracids Incisoscutum, Compagopiscis and Eastmanosteus. Anteriorly, these muscles terminate inside the nuchal and paranuchal plates of the skull roof (Fig. 1B to C and figs $\mathrm{S} 1 \mathrm{H}$ and $\mathrm{K}$ to I). The levator capitis major extends posteriorly to the anterior edge of the median dorsal keel (Figs $1 \mathrm{~B}$ to $\mathrm{C}$ and 3D to F). Judging by the relative sizes of the muscles, head elevation was more powerful than head depression; this suggests a functional role in rapid jaw opening associated with prey capture by $\operatorname{suction}(9,10,23$, 24). 
The cucullaris of eubrachythoracids inserts on the inner face of the dermal shoulder girdle (fig. S1M to N) and its proximal end lies in a hollow on the inner face of the skull roof, previously identified as the cucullaris fossa on positional criteria $(6)$.

In the holocephalan Callorhinchus milii, the cucullaris originates from a fossa on the posterior part of the neurocranium (fig. S3) and has several areas of insertion on the pectoral girdle. From dorsal to ventral, it inserts onto the rostral edge of the pectoral girdle, wraps round and inserts on the lateral face of the girdle, and finally inserts by means of a tendon onto a process at the base of the pectoral girdle. In arthrodires, the muscle is observed on the internal face of the ADL plate (Fig. 3J) and there is a small pit on the anterior face of the ADL, which is possibly the site for a tendon attachment. Thus the position and attachment of the eubrachythoracid cucullaris muscle appears in most respects similar to that found in holocephalans. The Gogo eubrachythoracids have unossified and thus unpreserved braincases, but in arthrodires with ossified braincases, such as buchanosteids, the braincase walls bear muscle attachment scars (fig. S1E). It is thus likely that the cucullaris of the Gogo eubrachythoracids originated on the braincase wall medial to the cucullaris fossa.

In extant gnathostomes the connective tissue of the cucullaris muscle incorporates pharyngeal neural crest (13) and its muscle cells are either derived mainly from the anterior somites $(5,25-28))$, from the occipital lateral plate mesoderm (29) or from the head mesoderm (Patel, conference communication). This issue is hotly debated but current consensus is that the cucullaris is a somite derivative.

Comparison between the transversus abdominis muscle of eubrachythoracids and the clasper and pelvic musculature in Callorhinchus milii.

In the eubrachythoracids, areas of transverse muscle fibres are found as far anteriorly as the inner face of the anterior median ventral plate, and can be seen in dorsal view through the nuchal gap of Eastmanosteus (Fig. 3b). Posteriorly, the transverse fibres end immediately anterior to the pelvis, which is equipped with claspers in males (30). This suggests the possibility that the muscles could be specifically for movement of the clasper. In order to test this hypothesis we dissected the pelvic musculature of a male elephant shark (holocephalan), Callorhinchus milii. The morphology of the pelvic fin and clasper in this fish is in many respects similar to that of that of eubrachythoracids, and the claspers are believed to be homologous (24).

We find no equivalent of the eubrachythoracid transversus abdominis in the elephant shark (fig S4). Two large muscles, the abdominal adductor (yellow) and pubic protractor (green), occupy part of the corresponding region in both males and females, but the muscle fibres for these muscles are oriented in an anteroposterior direction. The clasper muscles of males have the same anteroposterior orientation, with the exception of the obliquely oriented clasper obturator, which is positioned on the clasper itself.

Callorhinchus thus lacks a homologue of the eubrachythoracid transversus abdominis, notwithstanding the morphological similarity of the pelvis and clasper in chondrichthyans and eubrachythoracids. This in turn suggests that the transversus abdominis did not primarily serve to move the pelvis or clasper. 

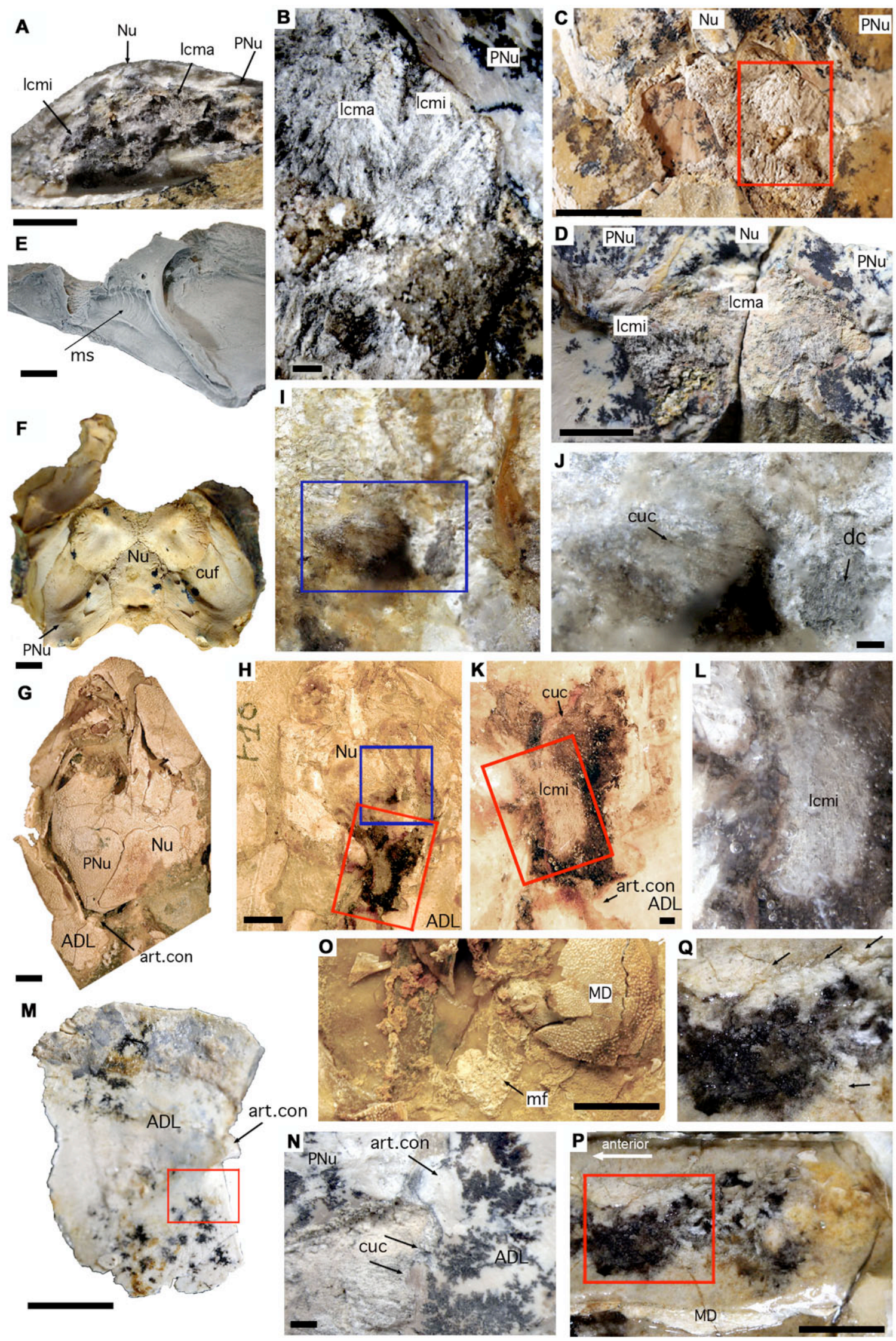


\section{Fig. S1.}

Nuchal gap musculature. A, Compagopiscis (WAM 11.12.5) posterior view of transverse break between head and trunk armour showing muscles under headshield. B, Compagopiscis (WAM 11.10.12) close up dorsal view of levator capitis minor and part of levator capitis major. $\mathbf{C}$, same specimen, dorsal view of levator capitis minor and levator capitis major; red box indicates area shown in B. D, same specimen, counterpart showing detail of levator capitis major on the internal surface of the nuchal plate. $\mathbf{E}$, internal posterolateral view of the skull and braincase (right side) of a buchanosteid placoderm showing muscle scars on the perichondral bone anterior to the cucullaris fossa. F, Internal view of the headshield of Eastmanosteus indicating the position of the cucullaris fossa. G, Incisoscutum (WAM 85.9.668) dorsal view of the head shield. H, internal view of same specimen showing nuchal gap muscles; red box enlarged in $\mathbf{K}$, further enlargement in $\mathbf{L}$; blue box enlarged in $\mathbf{I}$, further enlargement in $\mathbf{J}$. $\mathbf{M}$, Compagopiscis (WAM 86.9.673) Internal view of the anterior dorsolateral plate; red box indicates area where the cucullaris muscle extends onto the plate. N, Compagopiscis (WAM 11.12.5) cucullaris muscle extending from the paranuchal plate to the anterior dorsolateral plate. O. Incisoscutum ritchiei (WAM 95.1.1 juvenile) musculature from under the median dorsal plate. P, Compagopiscis (WAM 12.9.1) extent of the levator capitis major visible through the dermal bone surface of the median dorsal plate; red box enlarged in Q. Scale bars for A, C-H, M, O-P; $10 \mathrm{~mm}$; for B, J-K, N 1 mm. ADL, anterior dorsolateral plate; art.con, articular condyle of dermal neck joint; cuc, cucullaris muscle cuf, cucullaris fossa; dc dorsal branchial constrictor; MD, Median dorsal plate; mf, muscle fibres; ms, muscle scars; $\mathrm{Nu}$, Nuchal plate; lcma, levator capitis major; lcmi, levator capitis minor; $\mathrm{PNu}$, paranuchal plate. Arrows without labels indicate muscle fibre direction. 

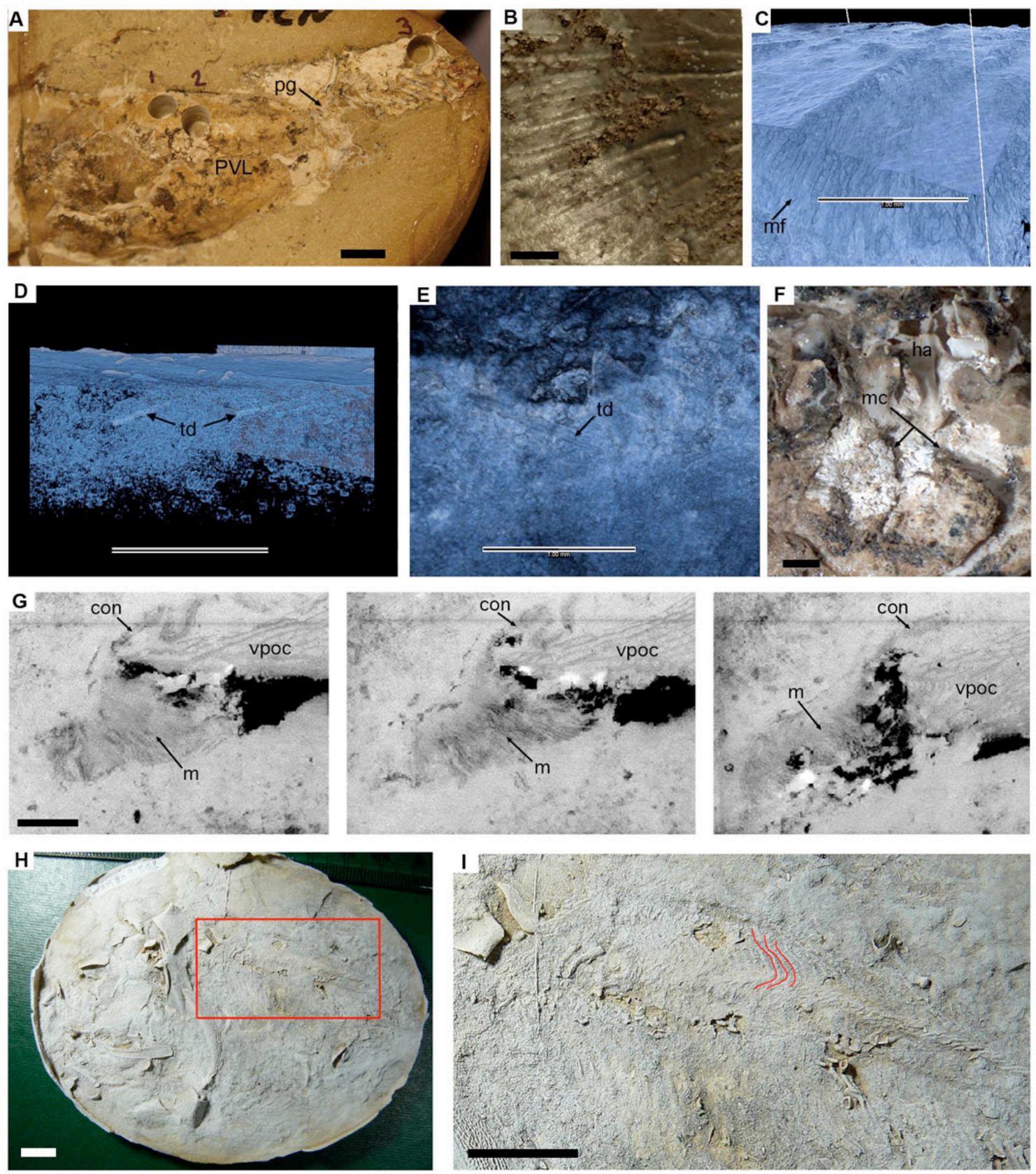


\section{Fig. S2}

Skin and musculature of the tail. A, Compagpopiscis croucheri (WAM 03.3.24), showing location of $\mu \mathrm{CT}$ sample cores in body $(1,2)$ and tail $(3)$. B, $\mu \mathrm{CT}$ model of body infill with natural mold of the internal surface of the bone, from sample site 1 in (A). C, individual muscle fibres from beneath the posterior ventrolateral plate. $\mathrm{D}-\mathrm{E}$, tendons beneath the epithelium from sample site 3 in (A) F, Incisoscutum ritchiei (NHM P57640) showing one muscle block straddling two vertebrae. $\mathrm{G}$, Three images from the PPC-SR $\mu \mathrm{CT}$ scan of Compagopiscis (WAM12.8.1), showing the ventral postocular process and the attached muscle. H, Australolepis gardineri (WAM 86.9.662), a ptyctodont with well-preserved trunk musculature. I, Close-up of region indicated by red box in $(\mathrm{H})$, showing dorsal half of myocommata (highlighted in red); the curvature is very shallow compared to recent chondrichthyans or bony fishes. Scale bars, for A, H-I, $10 \mathrm{~mm}$; for B-G, $1 \mathrm{~mm}$. con, connective tissue of muscle; ha, haemal arch; m, muscle; mf, mc, myocomma; muscle fibres; pg, pelvic girdle; PVL, posterior ventrolateral plate; td, tendon; vpoc, ventral postocular process. 

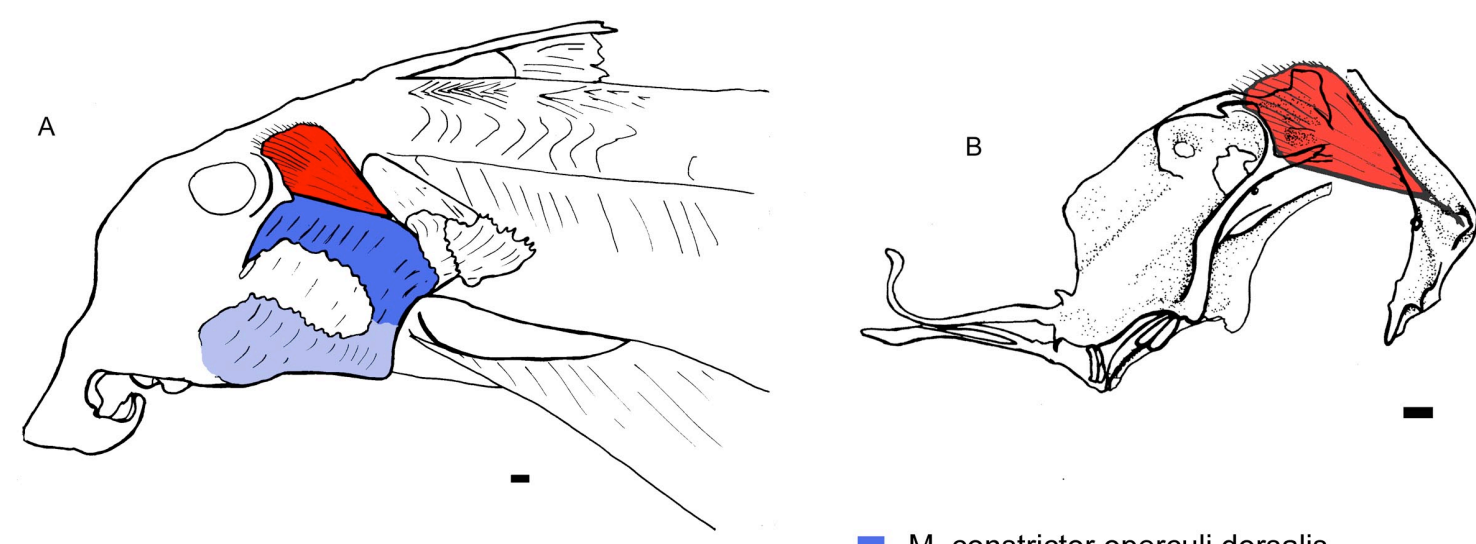

M. constrictor operculi dorsalis

M. constrictor operculi ventralis

Cucullaris

\section{Fig. S3}

Positioning and attachment area of the cucullaris muscle in the extant holocephalan Callorhinchus milii. A) Skinned whole specimen showing the cucullaris in place originating behind the orbit and inserting on the shoulder girdle underneath the mandibular constrictor dorsalis. B) Skeletonised specimen showing the origin and insertion points of the cucullaris on the cucullaris fossa and the posterior pectoral process respectively. Drawn from photos of the dissected specimens. Scale bars: $1 \mathrm{~cm}$. 


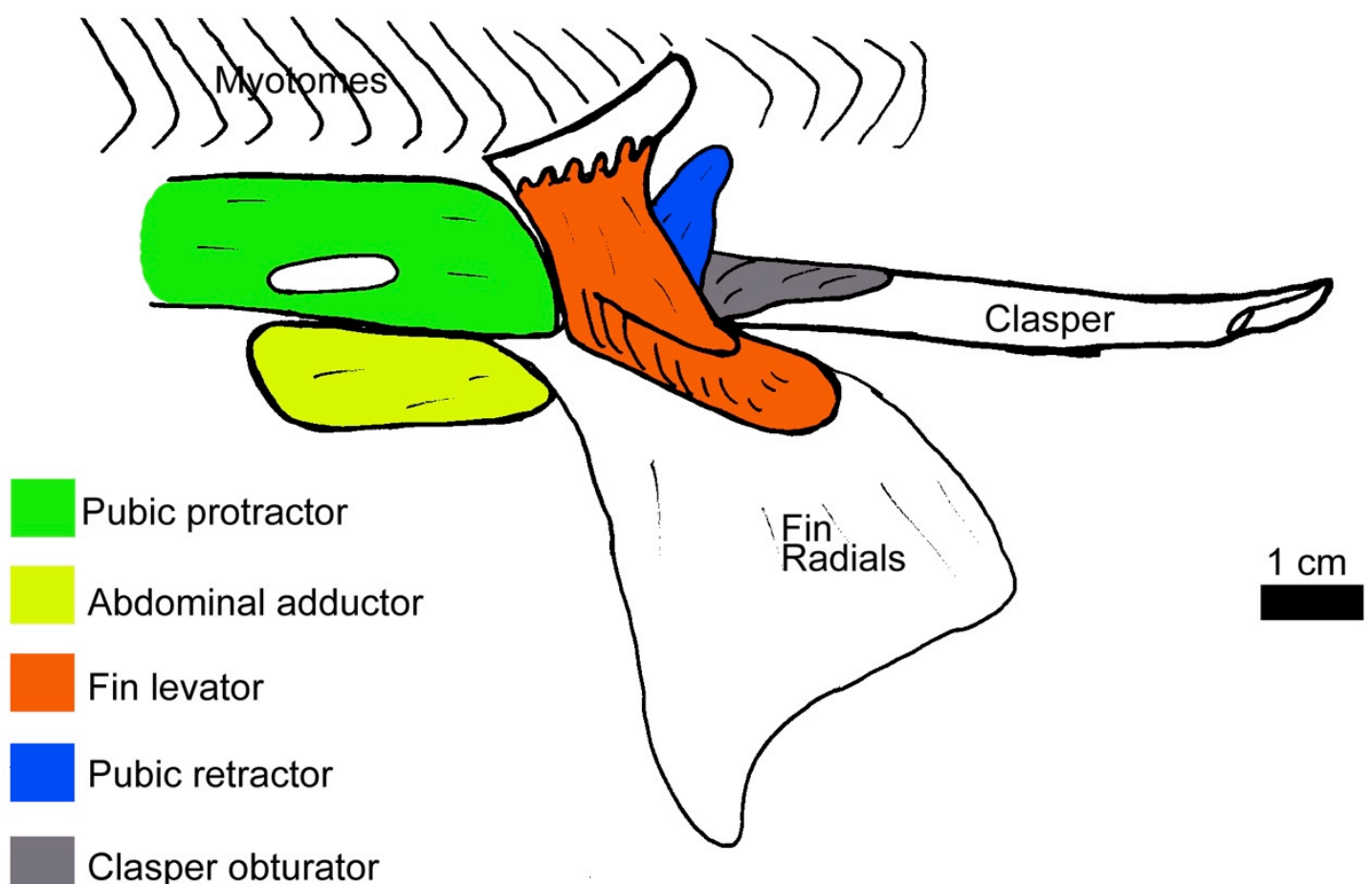

Fig. 54

Pelvic musculature in Callorhinchus milii, dorsal surface of the fin. Both male and females display the same pelvic muscle arrangement. Fibres of the pubic protractor and abdominal adductor are arranged anteroposteriorly. In males, the fibres of the clasper musculature follow the same anteroposterior orientation except for the clasper obturator, where the fibres are arranged obliquely to the body musculature. Black lines indicate muscle fibre orientation. 


\section{Movie S1}

Scroll-through movie of PPC-SR $\mu \mathrm{CT}$ scan showing the levator arcus palatini attached to the ventral postocular process of Compagopiscis (WAM12.8.1). Same scan as images in fig. S2G. 